\title{
Sarcopenia Is Associated With Impaired Overall Survival After Gastrectomy for Elderly Gastric Cancer
}

\author{
KAZUYOSHI YAMAMOTO ${ }^{1,2}$, MOTOHIRO HIRAO ${ }^{2}$, KAZUHIRO NISHIKAWA $^{2}$, TAKESHI OMORI ${ }^{1}$, \\ YOSHITOMO YANAGIMOTO ${ }^{1}$, NAOKI SHINNO ${ }^{1}$, KEIJIRO SUGIMURA $^{1}$, HIROSHI MIYATA ${ }^{1}$, \\ HIROSHI WADA ${ }^{1}$, HIDENORI TAKAHASHI ${ }^{1}$, MASAYOSHI YASUI ${ }^{1}$, MASAYUKI OHUE ${ }^{1}$, \\ MASAHIKO YANO ${ }^{1}$, KAZUMASA FUJITANI $^{3}$ and TOSHIMASA TSUJINAKA ${ }^{4}$ \\ ${ }^{1}$ Department of Gastroenterological Surgery, Osaka International Cancer Institute, Osaka, Japan; \\ ${ }^{2}$ Department of Surgery, National Hospital Organization Osaka National Hospital, Osaka, Japan; \\ ${ }^{3}$ Department of Surgery, Osaka General Medical Center, Osaka, Japan; \\ ${ }^{4}$ Department of Surgery, Kaizuka City Hospital, Osaka, Japan
}

\begin{abstract}
Background/Aim: The aim of this study was to evaluate the impact of sarcopenia diagnosed by the European Working Group on Sarcopenia in Older People (EWGSOP) algorithm on long-term outcome after gastrectomy. Patients and Methods: A total of 90 elderly gastric cancer patients without distant metastasis aged 65 years or older who underwent gastrectomy at the Osaka National Hospital between July 2012 and January 2015 were included in the current analysis. Results: The sarcopenic group $(n=19)$ had a poorer overall survival $(O S)(p<0.0001)$ compared to the non-sarcopenic group $(n=79)$. OS after recurrence was also worse in the sarcopenic group. Multivariate analysis indicated that sarcopenia was an independent risk factor for worse OS after gastrectomy (hazard ratio $(H R)=2.92 ; 95 \%$ confidence interval $(C I)=1.15-7.75 ; p=0.025)$, along with $N$ stage $\geq 2$, age $\geq 75$ years, and presence of severe postoperative complications. Conclusion: Sarcopenia is a potential target for preoperative intervention in elderly gastric cancer patients to improve prognosis after gastrectomy. (UMINCTR: R000041532)
\end{abstract}

Surgery is the most effective type of treatment for gastric cancer. The proportion of elderly patients with gastric cancer is increasing (1). Aging itself is considered a risk factor for operative mortality according to a risk model for gastrectomy based on data from a Japanese nationwide internet-based

Correspondence to: Kazuyoshi Yamamoto, MD, Ph.D., Department of Gastroenterological Surgery, Osaka International Cancer Institute, 3-169 Otemae, Chuo-ku, 541-8567 Osaka, Japan. Tel: +81 669451181, Fax: +81 669451900, e-mail: kazuyoshi0208yamamoto@yahoo.co.jp

Key Words: Elderly, gastrectomy, gastric cancer, prognosis, sarcopenia. database (2, 3). Appropriate risk estimation for elderly gastric cancer patients is important for improving surgical outcomes.

In 1989, sarcopenia was defined by Rosemberg as attenuation of muscle mass and muscle strength or physical performance associated with aging (4). The relationship between sarcopenia and treatment outcomes after surgery for gastrointestinal malignancies has been actively examined. A number of studies have demonstrated the negative impact of sarcopenia on short-term and long-term outcomes after surgery $(5,6)$. In terms of gastric cancer surgery, we previously reported (7) that among 99 elderly gastric cancer patients aged 65 years or older undergoing gastrectomy, 21 $(21.2 \%)$ were diagnosed with sarcopenia according to the algorithm proposed by the European Working Group on Sarcopenia in Older People (EWGSOP) (8). In addition, sarcopenia was identified as an independent risk factor for severe postoperative complications of Clavien-Dindo (CD) classification (9) grade III or higher, particularly nonsurgical complications such as pneumonia.

Several studies have investigated the negative impact of sarcopenia on survival (10), but the results were controversial (11) and diagnostic criteria and cut-off values for sarcopenia varied among them. Some studies determined sarcopenia only by measuring skeletal muscle from preoperative computed tomography (CT) images before cancer surgery (12). Cintosun et al. (13) have pointed out the limitations of such definitions and argued that muscle strength and definition of muscle function are important components of sarcopenia. The relationship between sarcopenia diagnosed using the original EWGSOP (8) algorithm with all of the criteria (e.g. muscle mass, muscle strength, and physical performance) and prognosis that includes stage stratification has not been fully investigated and will attract great interest. 
The purpose of this study was to investigate the impact of sarcopenia diagnosed using the authoritative criteria in the EWGSOP algorithm on long-term outcomes after gastrectomy in elderly gastric cancer patients.

\section{Patients and Methods}

Screening for sarcopenia. Preoperative screening for sarcopenia was based on diagnostic criteria from the EWGSOP algorithm (8) using gait speed $(\mathrm{m} / \mathrm{sec})$, handgrip strength $(\mathrm{kg})$, and skeletal muscle mass index (SMI, $\mathrm{kg} / \mathrm{m}^{2}$ ). Gait speed was calculated by dividing the distance of 4 meters by the time required $(\mathrm{m} / \mathrm{sec})$. A cut-off of 0.8 $\mathrm{m} / \mathrm{sec}$ was used to indicate the risk for sarcopenia. Handgrip strength was tested twice in each hand by a hand dynamometer, and the average of four values $(\mathrm{kg})$ was analyzed. A cut-off of $30 \mathrm{~kg}$ for men and $20 \mathrm{~kg}$ for women was employed to identify the risk for sarcopenia. SMI was calculated by dividing absolute skeletal muscle mass $(\mathrm{kg})$ measured using a bioimpedance analysis (BIA) with a multifrequency body composition analyzer, InBody 720 (Biospace) by height ${ }^{2}$. A cut-off of $8.87 \mathrm{~kg} / \mathrm{m} 2$ for men and $6.42 \mathrm{~kg} / \mathrm{m}^{2}$ for women was used based on EWGSOP recommendations.

Patients. Patients for the current analysis were the same patients as in our previous report (7). Between July 2012 and January 2015, a total of 99 consecutive gastric cancer patients aged 65 years or older underwent gastrectomy at Osaka National Hospital (ONH). Among 99 patients, 21 patients $(21.2 \%)$ were diagnosed with sarcopenia according to the EWGSOP algorithm (8). We excluded two patients from the sarcopenic group and seven patients from the nonsarcopenic group because they had synchronous distant metastases (M1). The remaining 90 patients (19 sarcopenic and 71 nonsarcopenic patients) who underwent R0 resection were included in the survival analysis of the current study (Figure 1).

Evaluation of clinical outcomes. Clinicopathological factors were compared between the sarcopenic $(n=19)$ and non-sarcopenic $(n=71)$ groups. Nutritional status was assessed using the preoperative serum albumin levesl (g/dl) and Onodera's Prognostic Nutritional Index (PNI) $(14,15)$ calculated as $10 \times$ serum albumin $(\mathrm{g} / \mathrm{dl})+0.005 \times$ total lymphocyte count $(/ \mathrm{mm} 3)$. Gastric cancer staging was based on the Japanese Classification of Gastric Carcinoma, third English edition (16). Postoperative surgical complications were graded according to the CD classification system (9) and severe complications were defined as CD grade IIIa or higher. In terms of adjuvant chemotherapy, S-1 [ACTS-GC trial (17) regimen] was recommended for pathological (p) stage II/III gastric cancer except for pT3N0 and pT1 disease by the 2014 gastric cancer treatment guideline from the Japanese Gastric Cancer Association (18). The induction rate for postoperative adjuvant chemotherapy in target patients was also compared between the two groups.

Follow-up. All 90 patients were followed in the OHN outpatient clinic after surgery with the goal of monitoring for recurrence according to the 2014 gastric cancer treatment guidelines from the Japanese Gastric Cancer Association (18). Patients received medical examinations, blood testing including tests for tumor markers such as carcinoembryonic antigen and carbohydrate antigen 19-9, and CT at least every 6 months until 5 years after surgery or when recurrence was confirmed. Treatment strategy and post-recurrence regimens were also compared between the two groups. Recurrence and survival data

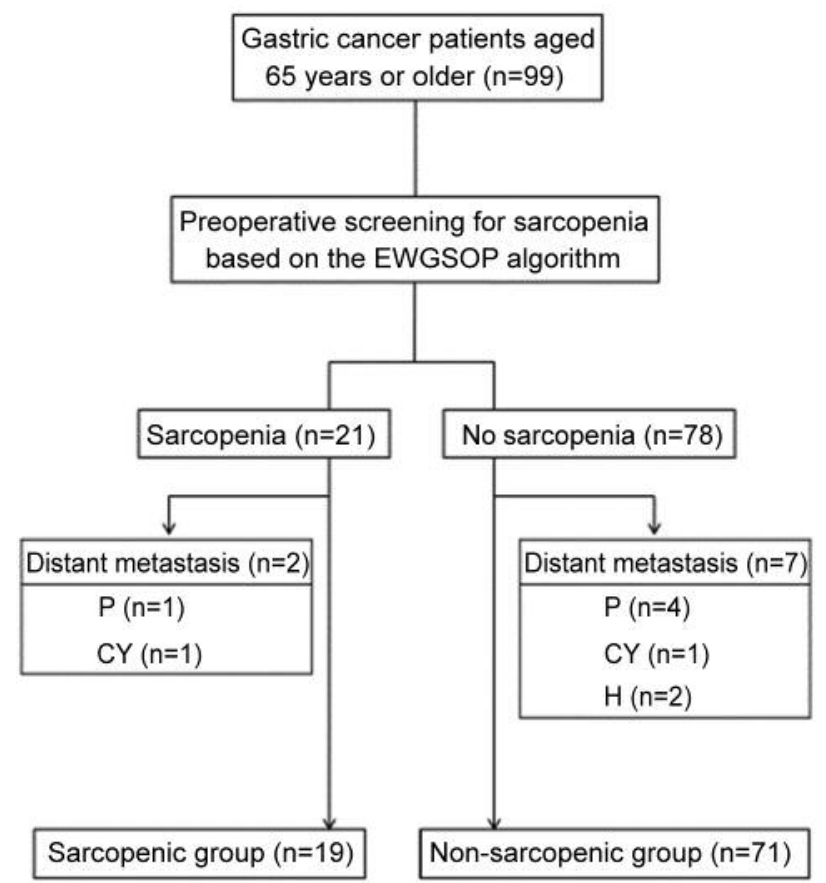

Figure 1. Study flow chart. CY: Peritoneal lavage cytology positive for carcinoma cells; EGWSOP: European Working Group on Sarcopenia in Older People; H: hepatic metastasis; P: peritoneal metastasis.

of enrolled patients were acquired from medical records. There were no enrolled patients lost to follow-up within 3 years of surgery.

Statistical analysis. We expressed continuous variables as medians (range). Fisher's exact test was used to compare binary variables. The Mann-Whitney $U$-test was used to compare continuous variables. Recurrence-free survival (RFS) was defined as the time from surgery to the first evidence of clinical recurrence of gastric cancer. Overall survival (OS) was defined as the time from surgery to death from any cause. Cancer-specific survival (CSS) was defined as the time from surgery until death from gastric cancer. Non-cancer-specific survival (non-CSS) was defined as the time from surgery to death from causes other than gastric cancer. OS after recurrence, which was defined as time from recurrence to death from any cause, was also examined between the two groups among patients who developed recurrence. Survival curves for RFS, OS, CSS, non-CSS, and OS after recurrence were estimated using the Kaplan-Meier method and compared using the log-rank test. Cox proportional hazard models were used in univariate and multivariate analyses of OS. $p$-Values $<0.05$ were considered statistically significant. Statistical analysis was conducted using JMP ${ }^{\circledR}$ software (SAS Institute, Cary, NC, USA).

\section{Results}

Patient characteristics. Clinicopathological and oncological factors of the sarcopenic $(n=19)$ and non-sarcopenic $(n=71)$ patients are shown in Table I. A higher proportion of sarcopenic patients were male $(89.5 \%$ vs. $62.0 \%, p=0.014)$. Sarcopenic patients had a lower body mass index (BMI) (18.9 
Table I. Clinicopathological factors in sarcopenic and non-sarcopenic patients.

\begin{tabular}{|c|c|c|c|}
\hline & $\begin{array}{l}\text { Sarcopenic } \\
\quad(n=19)\end{array}$ & $\begin{array}{l}\text { Non-sarcopenic } \\
\quad(\mathrm{n}=71)\end{array}$ & $p$-Value \\
\hline Age (years) & $78(67-83)$ & $75(66-91)$ & 0.094 \\
\hline Gender, n (\%) & & & 0.014 \\
\hline Male & $17(89.5)$ & $44(62.0)$ & \\
\hline Female & $2(10.5)$ & $27(38.0)$ & \\
\hline BMI $\left(\mathrm{kg} / \mathrm{m}^{2}\right)$ & $18.9(15.6-26.9)$ & $22.7(16.8-32.2)$ & 0.0026 \\
\hline Serum albumin level (g/dl) & $4.3(2.3-4.9)$ & $4.1(3.0-4.7)$ & 0.50 \\
\hline PNI & $51.2(29.3-64.9)$ & $50.3(37.4-62.1)$ & 0.83 \\
\hline ASA-PS, n (\%) & & & 0.69 \\
\hline $1-2$ & $15(78.9)$ & $53(74.7)$ & \\
\hline 3 & $4(21.1)$ & $18(25.4)$ & \\
\hline Surgical approach, n (\%) & & & 0.0056 \\
\hline Open & $18(94.7)$ & $47(66.2)$ & \\
\hline Laparoscopic & $1(5.3)$ & $24(33.8)$ & \\
\hline Procedure, n (\%) & & & 0.77 \\
\hline TG & $6(31.6)$ & $25(35.2)$ & \\
\hline Non-TG & $13(68.4)$ & $46(64.8)$ & \\
\hline Lymph node dissection, n (\%) & & & 0.33 \\
\hline D0-1 & $5(26.3)$ & $27(38.0)$ & \\
\hline D2 or higher & $14(73.7)$ & $44(62.0)$ & \\
\hline Operative time (min) & $291(172-530)$ & $281(141-561)$ & 0.67 \\
\hline Operative blood loss (ml) & $210(50-2900)$ & $160(10-1450)$ & 0.029 \\
\hline Severe postoperative complications ${ }^{\dagger}, \mathrm{n}(\%)$ & & & 0.0083 \\
\hline Yes & $6(31.6)$ & $5(7.0)$ & \\
\hline No & $13(68.4)$ & $66(93.0)$ & \\
\hline Pathological tumor depth, $\mathrm{n}(\%)$ & & & 0.0012 \\
\hline $\mathrm{T} 3-$ & $12(63.2)$ & $17(23.9)$ & \\
\hline T1a-2 & $7(36.8)$ & $54(76.1)$ & \\
\hline Pathological lymph node metastasis, n (\%) & & & 0.032 \\
\hline N2- & $9(47.4)$ & $16(22.5)$ & \\
\hline $\mathrm{N} 0-1$ & $10(52.6)$ & 77.5 & \\
\hline Neoadjuvant chemotherapy, n (\%) & & & 0.62 \\
\hline Yes & $1(5.3)$ & $2(2.8)$ & \\
\hline No & $18(94.7)$ & $69(97.2)$ & \\
\hline Adjuvant chemotherapy, $\mathrm{n}(\%)^{\ddagger}$ & & & 0.35 \\
\hline Yes & $6(54.5)$ & $17(70.8)$ & \\
\hline No & $5(45.5)$ & $7(29.2)$ & \\
\hline
\end{tabular}

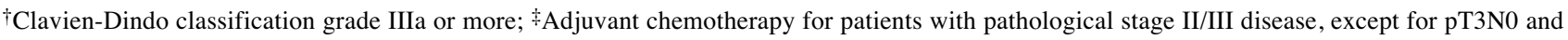
pT1. Gastric cancer staging based on the Japanese Classification of Gastric Carcinoma, third English edition. ASA-PS: American Society of Anesthesiologists physical status; BMI: body mass index; Non-TG: Non-total gastrectomy (includes distal gastrectomy and proximal gastrectomy); PNI: Prognostic Nutritional Index, calculated as $10 \times$ serum albumin $(\mathrm{g} / \mathrm{dl})+0.005 \times$ total lymphocyte count $\left(/ \mathrm{mm}^{3}\right)$; TG: total gastrectomy.

vs. $\left.22.7 \mathrm{~kg} / \mathrm{m}^{2}, p=0.002\right)$ than non-sarcopenic patients. In terms of nutritional parameters, there were no differences in preoperative serum albumin levels and PNI between the two groups. The open approach was used more frequently $(94.7 \%$ vs. $66.2 \%, p=0.0056)$ in sarcopenic patients because sarcopenic patients had more advanced disease based on the Japanese Classification of Gastric Carcinoma, third English edition (16). The rate of total gastrectomy and the extent of lymph node dissection were comparable between the two groups. As in our previous report, the incidence of severe postoperative complications of CD classification grade IIIa or higher was significantly higher in sarcopenic than non-
Table II. First treatment after recurrence.

\begin{tabular}{lccc}
\hline & $\begin{array}{c}\text { Sarcopenic } \\
(\mathrm{n}=8)\end{array}$ & $\begin{array}{c}\text { Non-sarcopenic } \\
(\mathrm{n}=7)\end{array}$ & $p$-Value \\
\hline Metastasectomy $^{\dagger}$ & 0 & $3^{*}$ & 0.13 \\
Chemotherapy $_{\text {Platinum-based doublet }}$ & 7 & 3 & \\
Taxane \pm ramucirmab & 1 & 2 & \\
S-1 monotherapy & 1 & 1 & \\
None & 1 & 0 & \\
\hline
\end{tabular}

$\dagger$ Metastasectomy was performed for lung metastasis in two patients and rectum metastasis in one patient. 
a

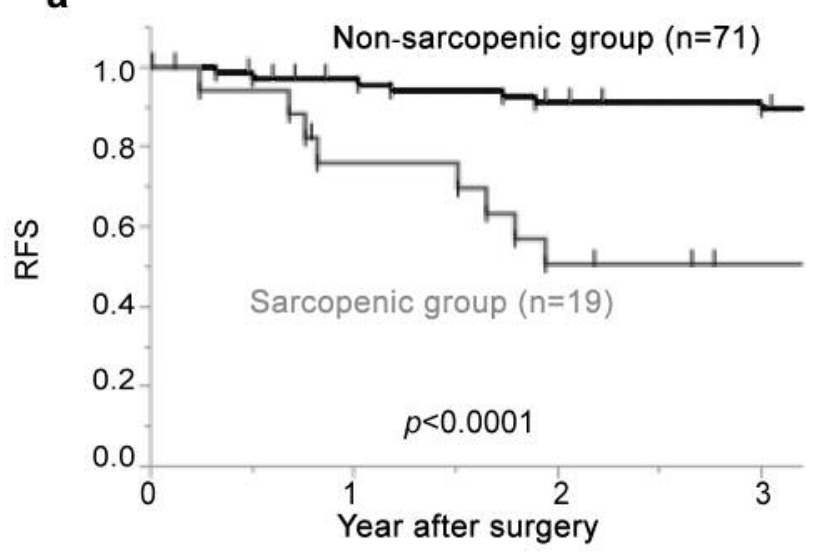

C

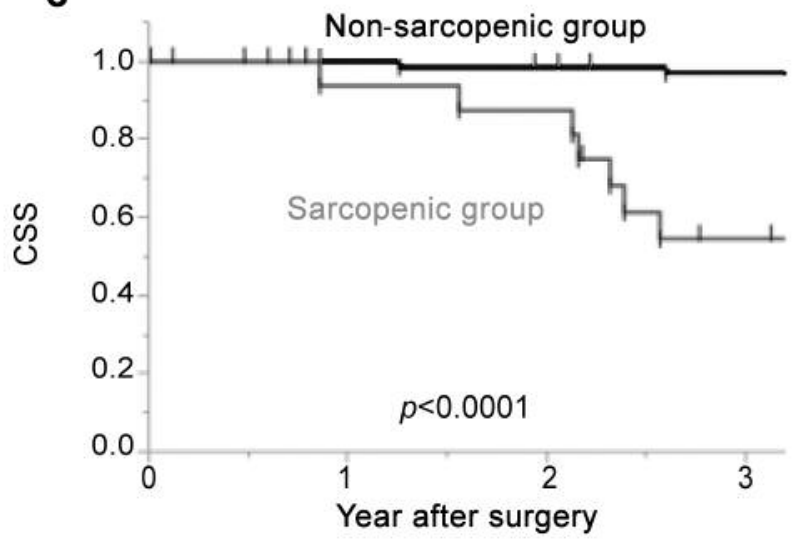

b

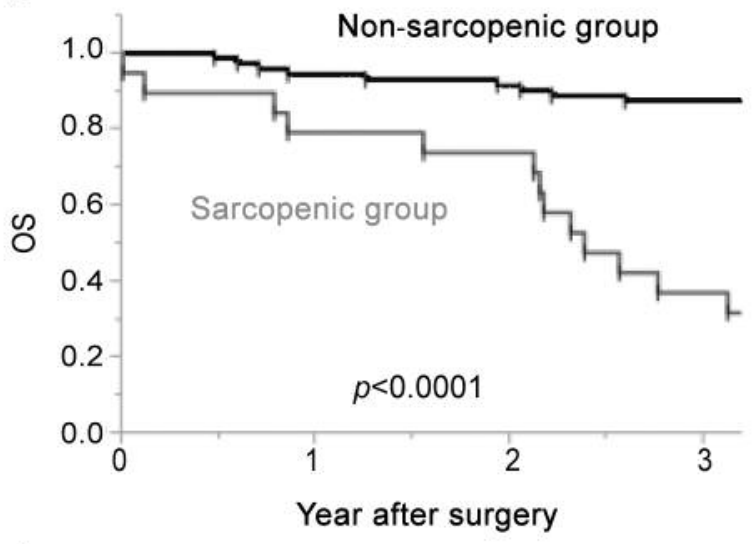

d

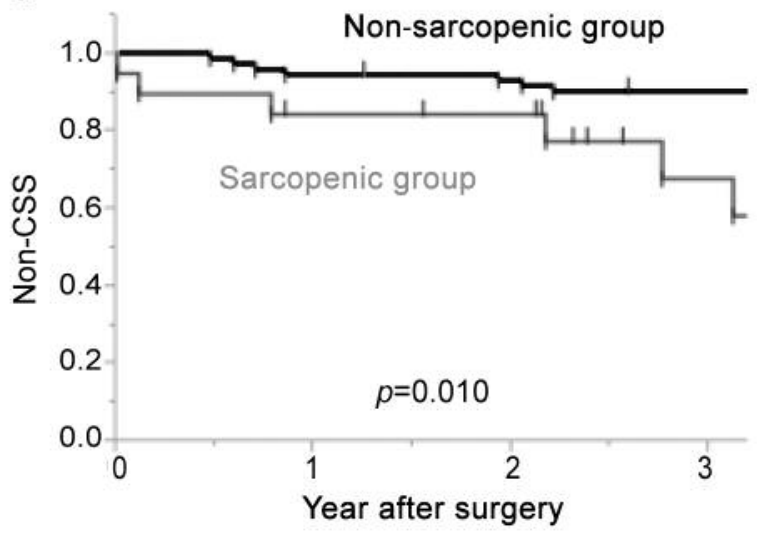

Figure 2. Recurrence-free survival, overall survival, cancer-specific survival, and non-cancer-specific survival in sarcopenic and non-sarcopenic patients. a) Recurrence-free survival after surgery; b) Overall survival after surgery; c) Cancer-specific survival after surgery; d) Non-cancerspecific survival after surgery. The blue line indicates the survival curve of sarcopenic patients. The red line indicates the survival curve of nonsarcopenic patients. CSS: Cancer specific survival; Non-CSS: non-cancer-specific survival; OS: overall survival; RFS: recurrence-free survival.

sarcopenic patients $(31.6 \%$ vs. $7.0 \%, p=0.0083)$. The proportion of patients who received postoperative adjuvant chemotherapy among patients with $\mathrm{p}$ Stage II/III disease except for pT3N0/pT1 disease tended to be lower in the sarcopenic group than in the non-sarcopenic group $(54.5 \% \mathrm{vs}$. $70.8 \%)$; however, this difference did not reach statistical significance $(p=0.35)$.

Recurrence-free survival, overall survival, cancer-specific survival, and non-cancer-specific survival after gastrectomy. Survival analysis was performed after a median observation period of 55 months. There were no censored cases within 3 years after surgery in the OS analysis. As shown in Figure 2, RFS $(p<0.0001)$ and OS $(p<0.0001)$ were significantly worse in sarcopenic patients. Furthermore, both CSS $(p<0.0001)$ and nonCSS $(p=0.011)$ were significantly worse in sarcopenic patients. The main causes of non-CSS were pneumonia $(n=8)$, cardiac event $(n=5)$, other cancers $(n=3)$, and renal dysfunction $(n=2)$.
Treatment strategy and OS after recurrence. Eight patients in the sarcopenic group and seven patients in non-sarcopenic group developed recurrence. The initial site of recurrence in the sarcopenic group was the peritoneum in five patients, liver in two patients, and distant lymph node in one patient. In the non-sarcopenic group, the initial site of recurrence was the peritoneum in two patients, liver in two patients, lung in two patients, and rectum in one patient. Treatment strategies after recurrence are shown in Table II. More aggressive treatments, such as metastasectomy for lung and rectum metastasis and chemotherapy using platinum-based doublet regimens, tended to be employed in non-sarcopenic patients. In contrast, chemotherapy using taxane or S-1 monotherapy tended to be administered to sarcopenic patients. One patient from each group did not receive any treatment after recurrence because of advanced age and patient refusal. Sarcopenic patients had significantly worse OS after recurrence than non-sarcopenic patients $(p=0.043)$. The 
Table III. Univariate and multivariate analysis of OS after surgery.

\begin{tabular}{|c|c|c|c|c|c|c|c|c|}
\hline & & \multirow[b]{2}{*}{$\mathrm{n}$} & \multicolumn{3}{|c|}{ Univariate } & \multicolumn{3}{|c|}{ Multivariate } \\
\hline & & & HR & $95 \% \mathrm{CI}$ & $p$-Value & HR & $95 \% \mathrm{CI}$ & $p$-Value \\
\hline \multirow[t]{2}{*}{ Gender } & Male & 61 & 1.89 & $0.81-5.15$ & 0.15 & 0.97 & $0.34-2.96$ & 0.96 \\
\hline & Female & 29 & & & & & & \\
\hline \multirow[t]{2}{*}{ Age (years) } & $\geq 75$ & 50 & 2.60 & $1.15-6.63$ & 0.021 & 2.46 & $1.06-6.38$ & 0.036 \\
\hline & $<75$ & 40 & & & & & & \\
\hline \multicolumn{9}{|l|}{ EWGSOP } \\
\hline \multirow[t]{2}{*}{ Sarcopenia } & Yes & 19 & 6.81 & $3.16-14.99$ & $<0.0001$ & 2.92 & $1.15-7.75$ & 0.025 \\
\hline & No & 71 & & & & & & \\
\hline \multirow[t]{2}{*}{ Severe postoperative complications ${ }^{\dagger}$} & Yes & 11 & 5.17 & $2.12-11.47$ & 0.0007 & 9.47 & $2.97-31.52$ & 0.0002 \\
\hline & No & 79 & & & & & & \\
\hline \multirow[t]{2}{*}{ Tumor depth } & pT3- & 29 & 4.40 & $2.04-10.02$ & 0.0002 & 1.39 & $0.50-3.84$ & 0.52 \\
\hline & pT1-2 & 61 & & & & & & \\
\hline \multirow[t]{2}{*}{ Lymph node metastasis } & $\mathrm{pN} 2-$ & 25 & 3.93 & $1.83-8.61$ & 0.0005 & 6.69 & $2.23-21.67$ & 0.0010 \\
\hline & pN0-1 & 65 & & & & & & \\
\hline
\end{tabular}

$\dagger$ Clavien-Dindo classification grade IIIa or more. Gastric cancer staging based on the Japanese Classification of Gastric Carcinoma, third English edition. EWGSOP: European Working Group on Sarcopenia in Older People.

1-year survival rate was $50.0 \%$ in sarcopenic patients, compared with $71.4 \%$ in non-sarcopenic patients (Figure 3).

Impact of sarcopenia on OS after gastrectomy. To stratify by stage of gastric cancer, we used Cox proportional hazards models to investigate the impact of sarcopenia on OS after gastrectomy (Table III). M stage (pM1/pM0) was not used as covariate factor in this analysis because patients who had synchronous distant metastases (pM1) were excluded from this study and all eligible patients were M0. Age $\geq 75$ years, sarcopenia diagnosed based on the EWGSOP algorithm, presence of severe postoperative complications, T stage (pT3/pT1-2), and $\mathrm{N}$ stage (pN2-/pN0-1) were significant risk factors for worse OS in univariate analysis. Multivariate analysis demonstrated that sarcopenia diagnosed based on the EWGSOP algorithm (hazard ratio $(\mathrm{HR})=2.92 ; 95 \%$ confidence interval $(\mathrm{CI})=1.15-7.75 ; p=0.025)$ is an independent negative prognostic factor for OS after gastrectomy along with age, presence of severe postoperative complications, and $\mathrm{N}$ stage.

\section{Discussion}

Increasing age leads to reduced skeletal muscle mass, muscle strength, and physical performance. Sarcopenia (4), which is recognized as a risk factor for worse treatment outcomes in the elderly, will become a more important issue in a rapidly aging society. We investigated the clinical impact of sarcopenia on long-term outcomes after gastrectomy in elderly gastric cancer patients. In this study, the sarcopenic group included more male patients, had lower BMI and more advanced disease, and developed severe postoperative complications more frequently. Sarcopenic patients had

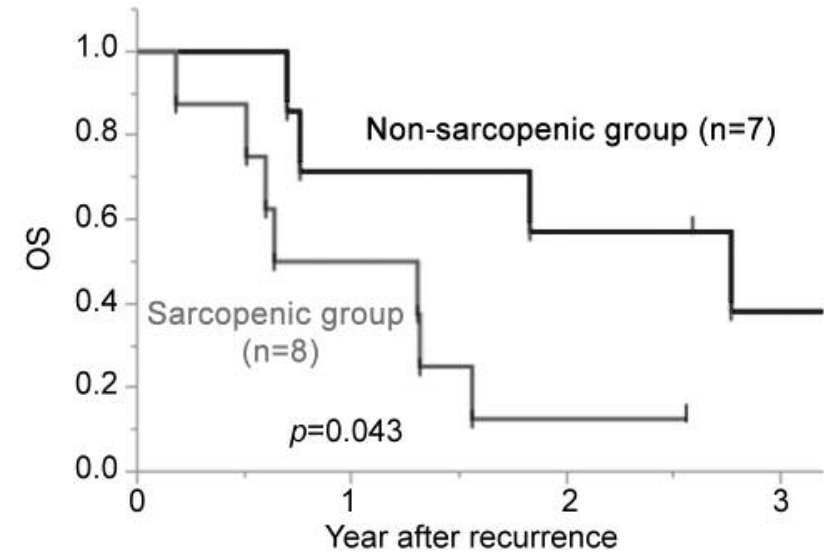

Figure 3. Overall survival after recurrence in sarcopenic $(n=8)$ and non-sarcopenic $(n=7)$ patients. OS: Overall survival.

worse RFS, OS, CSS, non-CSS, and OS after recurrence. Furthermore, multivariate analysis showed that sarcopenia is an independent risk factor for worse OS after gastrectomy in elderly gastric cancer patients.

Sarcopenia has recently received much attention from surgical oncologists. Many studies have investigated the relationship between sarcopenia and surgical outcomes after gastrectomy in elderly gastric cancer patients. The majority of these studies demonstrated that sarcopenia is associated with an increased risk of morbidity $(7,10)$, worse diseasespecific survival $(10,19)$, and higher risk of death from other causes (20) after gastrectomy. However, the definition and cutoff values for sarcopenia varied widely across studies (12); studies in which sarcopenia was diagnosed 
preoperatively based on evaluation of skeletal muscle mass, muscle strength, and physical performance were rare. We used the authoritative, well-described EWGSOP algorithm to diagnose sarcopenia in this study and examined the background characteristics and treatment strategies of sarcopenic and non-sarcopenic patients. We demonstrated the negative impact of sarcopenia on short-term and long-term outcomes using the same cohort.

Assessment of sarcopenia before gastric cancer surgery will provide useful information for risk stratification and choosing optimal treatment strategies. Based on the results of our previous report, we developed a preoperative exercise and nutritional support program (21) for elderly sarcopenic patients with gastric cancer to reduce the severity of postoperative complications. We have demonstrated that this preoperative program reduced the severity of sarcopenia to some extent, even with a limited duration of program participation. The current study provided some evidence that this program might improve prognosis after surgery.

However, the mechanisms underlying impaired prognosis after gastrectomy in sarcopenic patients with gastric cancer remain unclear. There are three possible explanations of worse prognosis after gastrectomy in sarcopenic patients with gastric cancer. First, the rate of severe postoperative complications was significantly higher in sarcopenic patients. Kubota et al. (22) indicated that postoperative complications that can cause prolonged inflammation are associated with worse prognosis. Second, sarcopenic patients might have impaired tolerance for gastric cancer treatment. In this study, the induction rate of postoperative adjuvant chemotherapy among target patients tended to be lower in sarcopenic group. Also, more invasive treatments such as metastasectomy or platinum-based doublet chemotherapy were not employed after recurrence in the sarcopenic group. Previous reports have indicated that decreased muscle mass is associated with discontinuation and decreased relative dose intensity of neoadjuvant (23) and palliative (24) chemotherapy for gastric cancer because the clearance of cytotoxic agents was reported to be dependent on lean body mass (25), not body surface area (BSA), which is the most important value for deciding on the dose of chemotherapy. Third, declines in skeletal muscle mass that characterize sarcopenia have been reported to be associated with declines in adaptive and innate immunity, including cancer immunity, through decreased expression of myokines and suppression of the development of natural killer lymphocytes (26). These factors mentioned above might reflect the combined negative impact of sarcopenia on prognosis.

There were some limitations in this study. The sample size was small and detailed evaluation of cancer immunity or expression of myokines in skeletal muscle was not performed. Treatment regimens after recurrence varied because of the observational nature of the study. Examination of cancer immunity and myokines related to skeletal muscle and tolerance of chemotherapy and prognosis of elderly patients will be challenging issues for future trials. Furthermore, prospective interventional trials will be necessary to evaluate the effect of preoperative exercise and nutritional support programs not only for reducing sarcopenia and severe postoperative complications, but also for improving prognosis.

In conclusion, our study suggests that sarcopenia is an independent risk factor for poor prognosis after gastrectomy in elderly gastric cancer patients.

\section{Conflicts of Interest}

The Authors declare that they have no conflicts of interest regarding this study.

\section{Acknowledgements}

The Authors wish to thank Yukiko Nagatsuma, Akiko Toriyama, Chihiro Nakahara, and Tamaki Nakayama, nationally registered dietitians at $\mathrm{ONH}$, for their assistance with diagnosing sarcopenia and for providing nutritional counseling after gastrectomy for patients enrolled in this study.

\section{Authors' Contributions}

Kazuyoshi Yamamoto designed the study and wrote the initial draft of the manuscript. Toshimasa Tsujinaka contributed to analysis and interpretation of data and assisted in the preparation of the manuscript. All other authors have contributed to data collection and interpretation, and critically reviewed the manuscript. All Authors approved the final version of the manuscript and agree to be accountable for all aspects of the work in ensuring that questions related to the accuracy or integrity of any part of the work are appropriately investigated and resolved.

\section{References}

1 Arai T, Esaki Y, Inoshita N, Sawabe M, Kasahara I, Kuroiwa K, Honma $\mathrm{N}$ and Takubo K: Pathologic characteristics of gastric cancer in the elderly: a retrospective study of 994 surgical patients. Gastric Cancer 7(3): 154-159, 2004. PMID: 15449203. DOI: $10.1007 / \mathrm{s} 10120-004-0285-4$

2 Kurita N, Miyata H, Gotoh M, Shimada M, Imura S, Kimura W, Tomita N, Baba H, Kitagawa Y, Sugihara K and Mori M: Risk model for distal gastrectomy when treating gastric cancer on the basis of data from 33,917 Japanese patients collected using a nationwide web-based data entry system. Ann Surg 262(2): 295303, 2015. PMID: 25719804. DOI: 10.1097/SLA.00000 00000001127

3 Watanabe M, Miyata H, Gotoh M, Baba H, Kimura W, Tomita N, Nakagoe T, Shimada M, Kitagawa Y, Sugihara K and Mori M: Total gastrectomy risk model: data from 20,011 Japanese patients in a nationwide internet-based database. Ann Surg 260(6): 1034-1039, 2014. PMID: 25072429. DOI: 10.1097/SLA. 0000000000000781

4 Rosenberg IH: Sarcopenia: origins and clinical relevance. J Nutr 127(5 Suppl): 990S-991S, 1997. PMID: 9164280. DOI: 10.1093/ jn/127.5.990S 
5 Ida S, Watanabe M, Yoshida N, Baba Y, Umezaki N, Harada K, Karashima R, Imamura Y, Iwagami S and Baba H: Sarcopenia is a predictor of postoperative respiratory complications in patients with esophageal cancer. Ann Surg Oncol 22(13): 4432-4437, 2015. PMID: 25862583. DOI: 10.1245/s10434-015-4559-3

6 Reisinger KW, van Vugt JL, Tegels JJ, Snijders C, Hulsewé KW, Hoofwijk AG, Stoot JH, Von Meyenfeldt MF, Beets GL, Derikx JP and Poeze M: Functional compromise reflected by sarcopenia, frailty, and nutritional depletion predicts adverse postoperative outcome after colorectal cancer surgery. Ann Surg 261(2): 345-352, 2015. PMID: 24651133. DOI: 10.1097/SLA. 0000000000000628

7 Fukuda Y, Yamamoto K, Hirao M, Nishikawa K, Nagatsuma Y, Nakayama T, Tanikawa S, Maeda S, Uemura M, Miyake M, Hama N, Miyamoto A, Ikeda M, Nakamori S, Sekimoto M, Fujitani $\mathrm{K}$ and Tsujinaka T: Sarcopenia is associated with severe postoperative complications in elderly gastric cancer patients undergoing gastrectomy. Gastric Cancer 19(3): 986-993, 2016. PMID: 26407875. DOI: 10.1007/s10120-015-0546-4

8 Cruz-Jentoft AJ, Baeyens JP, Bauer JM, Boirie Y, Cederholm T, Landi F, Martin FC, Michel JP, Rolland Y, Schneider SM, Topinková E, Vandewoude M, Zamboni $\mathrm{M}$ and European Working Group on Sarcopenia in Older People: Sarcopenia: European consensus on definition and diagnosis: Report of the European Working Group on Sarcopenia in Older People. Age Ageing 39(4): 412-423, 2010. PMID: 20392703. DOI: 10.1093/ ageing/afq034

9 Clavien PA, Barkun J, de Oliveira ML, Vauthey JN, Dindo D, Schulick RD, de Santibañes E, Pekolj J, Slankamenac K, Bassi C, Graf R, Vonlanthen R, Padbury R, Cameron JL and Makuuchi $\mathrm{M}$ : The Clavien-Dindo classification of surgical complications: five-year experience. Ann Surg 250(2): 187-196, 2009. PMID: 19638912. DOI: 10.1097/SLA.0b013e3181b13ca2

10 Kawamura T, Makuuchi R, Tokunaga M, Tanizawa Y, Bando E, Yasui $\mathrm{H}$, Aoyama $\mathrm{T}$, Inano $\mathrm{T}$ and Terashima $\mathrm{M}$ : Long-term outcomes of gastric cancer patients with preoperative sarcopenia. Ann Surg Oncol 25(6): 1625-1632, 2018. PMID: 29633095. DOI: $10.1245 / \mathrm{s} 10434-018-6452-3$

11 Tegels JJ, van Vugt JL, Reisinger KW, Hulsewé KW, Hoofwijk AG, Derikx JP and Stoot JH: Sarcopenia is highly prevalent in patients undergoing surgery for gastric cancer but not associated with worse outcomes. J Surg Oncol 112(4): 403-407, 2015. PMID: 26331988. DOI: 10.1002/jso.24015

12 Shen Y, Hao Q, Zhou J and Dong B: The impact of frailty and sarcopenia on postoperative outcomes in older patients undergoing gastrectomy surgery: a systematic review and metaanalysis. BMC Geriatrics 17(1): 188, 2017. PMID: 28826406. DOI: $10.1186 / \mathrm{s} 12877-017-0569-2$

13 Cintosun U, Tasci I and Safer VB: Computerized Tomographybased Diagnosis of Sarcopenia? Ann Surg 263(1): e4, 2016. PMID: 24866544. DOI: 10.1097/SLA.0000000000000774

14 Onodera T, Goseki N and Kosaki G: Prognostic nutritional index in gastrointestinal surgery of malnourished cancer patients. Nihon Geka Gakkai Zasshi 85(9): 1001-1005, 1984. PMID: 6438478

15 Migita K, Takayama T, Saeki K, Matsumoto S, Wakatsuki K, Enomoto K, Tanaka T, Ito M, Kurumatani N and Nakajima Y: The prognostic nutritional index predicts long-term outcomes of gastric cancer patients independent of tumor stage. Ann Surg Oncol 20(8): 2647-2654, 2013. PMID: 23463091. DOI: 10.1245/ s10434-013-2926-5
16 Japanese Gastric Cancer Association: Japanese classification of gastric carcinoma: 3rd English edition. Gastric Cancer 14(2): 101112, 2011. PMID: 21573743. DOI: 10.1007/s10120-011-0041-5

17 Sakuramoto S, Sasako M, Yamaguchi T, Kinoshita T, Fujii M, Nashimoto A, Furukawa H, Nakajima T, Ohashi Y, Imamura H, Higashino M, Yamamura Y, Kurita A, Arai K and ACTS-GC Group: Adjuvant chemotherapy for gastric cancer with S-1, an oral fluoropyrimidine. N Engl J Med 357(18): 1810-1820, 2007. PMID: 17978289. DOI: 10.1056/NEJMoa072252

18 Japanese Gastric Cancer Association: Japanese gastric cancer treatment guidelines 2014 (ver. 4). Gastric Cancer 20(1): 1-19, 2017. PMID: 27342689. DOI: 10.1007/s10120-016-0622-4

19 Kudou K, Saeki H, Nakashima Y, Edahiro K, Korehisa S, Taniguchi D, Tsutsumi R, Nishimura S, Nakaji Y, Akiyama S, Tajiri H, Nakanishi R, Kurashige J, Sugiyama M, Oki E and Maehara Y: Prognostic significance of sarcopenia in patients with esophagogastric junction cancer or upper gastric cancer. Ann Surg Oncol 24(7): 1804-1810, 2017. PMID: 28224363. DOI: $10.1245 / \mathrm{s} 10434-017-5811-9$

20 Kuwada K, Kuroda S, Kikuchi S, Yoshida R, Nishizaki M, Kagawa $S$ and Fujiwara T: Sarcopenia and comorbidity in gastric cancer surgery as a useful combined factor to predict eventual death from other causes. Ann Surg Oncol 25(5): 1160-1166, 2018. PMID: 29404820. DOI: 10.1245/s10434-018-6354-4

21 Yamamoto K, Nagatsuma Y, Fukuda Y, Hirao M, Nishikawa K, Miyamoto A, Ikeda M, Nakamori S, Sekimoto M, Fujitani K and Tsujinaka T: Effectiveness of a preoperative exercise and nutritional support program for elderly sarcopenic patients with gastric cancer. Gastric Cancer 20(5): 913-918, 2017. PMID: 28032232. DOI: 10.1007/s10120-016-0683-4

22 Kubota T, Hiki N, Sano T, Nomura S, Nunobe S, Kumagai K, Aikou S, Watanabe R, Kosuga T and Yamaguchi T: Prognostic significance of complications after curative surgery for gastric cancer. Ann Surg Oncol 21(3): 891-898, 2014. PMID: 24254205. DOI: $10.1245 / \mathrm{s} 10434-013-3384-9$

23 Tan BH, Brammer K, Randhawa N, Welch NT, Parsons SL, James EJ and Catton JA: Sarcopenia is associated with toxicity in patients undergoing neo-adjuvant chemotherapy for oesophago-gastric cancer. Eur J Surg Oncol 41(3): 333-338, 2015. PMID: 25498359. DOI: 10.1016/j.ejso.2014.11.040

24 Lee JS, Kim YS, Kim EY and Jin W: Prognostic significance of CT-determined sarcopenia in patients with advanced gastric cancer. PLoS One 13(8): e0202700, 2018. PMID: 30125312. DOI: 10.1371 /journal.pone. 0202700

25 Prado CM, Baracos VE, McCargar LJ, Reiman T, Mourtzakis M, Tonkin K, Mackey JR, Koski S, Pituskin E and Sawyer MB: Sarcopenia as a determinant of chemotherapy toxicity and time to tumor progression in metastatic breast cancer patients receiving capecitabine treatment. Clin Cancer Res 15(8): 2920-2926, 2009. PMID: 19351764. DOI: 10.1158/1078-0432.CCR-08-2242

26 Lutz CT and Quinn LS: Sarcopenia, obesity, and natural killer cell immune senescence in aging: altered cytokine levels as a common mechanism. Aging 4(8): 535-546, 2012. PMID: 22935594. DOI: $10.18632 /$ aging. 100482
Received May 26, 2019

Revised June 24, 2019

Accepted June 25, 2019 